$$
\begin{aligned}
& =\frac{E}{2 R}\left(1-\epsilon^{-R t / L}\right)-\frac{E}{2 R} \epsilon^{-R t / L}+\frac{E}{2 R} \\
& =E / R\left(1-\epsilon^{-R t / L}\right)
\end{aligned}
$$

Equation (17) was obtained with the aid of a table of definite integrals.

2. The current through an inductance and resistance in series, caused by suddenly impressing a continued sine wave of voltage may be obtained by substituting in equation (13) the values of $u$ and $v$ from equation (15). The result is

$$
\begin{gathered}
I=\frac{E}{R^{2}+P^{2} L^{2}}\left(P L \epsilon^{-R t / L}+R \sin P t\right. \\
-P L \cos P t)
\end{gathered}
$$

\title{
Energy Front of Electric Circuits
}

\author{
BY CHARLES P. STEINMETZ \\ Fellow, A. I. E. E. \\ Chief Consulting Engineer, General Electric Company, Schenectady, N. Y.
}

$\mathrm{A}^{\mathrm{s}}$

$\mathrm{S}$ there has been recently some discussion of the position of the energy front in an electric circuit, and as this discussion mainly consisted in quoting mathematical authorities against each other, it may be of interest to the reader who does not care to go into the mathematics of it, to discuss in non-mathematical terms the energy travel in the space surrounding a conductor carrying electric current.

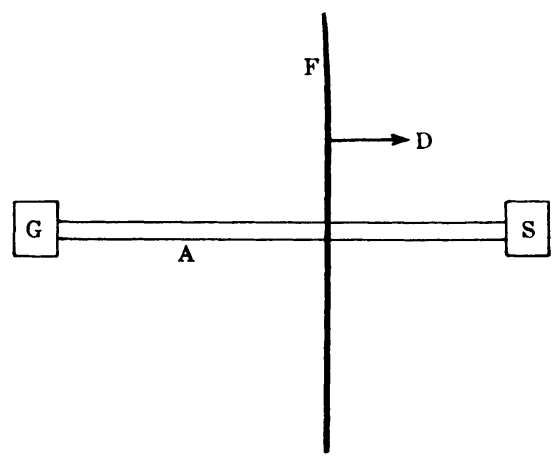

FIG. 1

It is obvious that there is no real disagreement between the mathematical conclusions, but the apparent disagreement is merely the result of misinterpretation.

Assume electric power is transmitted from a generating station $G$ over a transmission line $A$ into a receiving station $S$.

1. Let us first consider the ideal case of no energy loss, that is, assume a perfect conductor of zero resistance, and that all the energy sent out by the generating station $G$, arrives at the receiving station $S$. The electric field of the conductor and the energy carried by it must then move parallel to the conductor, as shown by the arrow $D$ in Fig. 1; that is, the wave front of the energy travel, or "energy front" is perpendicular to the conductor, as shown by $F$ in Fig. 1. This is obvious, as only the energy moving parallel with $A$ can arrive at $S$.

2. There is however inevitably some energy dissipated in the ohmic resistance of the conductor $A$. Physically, this appears as a slowing down of the speed of travel of the energy front in the conductor, behind that in free space ${ }^{1}$, so that the energy front in the conductor, $F$, in Fig. 2, lags behind the energy front $F_{0}$ in surrounding space. This means that the energy front near the conductor cannot remain perpendicular but bends back, as shown in Fig. 3, and the direction of energy travel near the conductor converges towards the conductor as seen by the arrows in Fig. 3.

3. On a perpendicular, the distance $a G$ Fig. 4, at some distance from the conductor $A$, is greater than the distance $b G$, near the conductor $A$. The energy front therefore cannot remain perpendicular to the conductor $A$ at greater distance from the conductor but must curve back, as shown by the dotted line in Fig. 4, so that the distances (along the stream lines) from the source of energy to the parts of the energy front in space are the same.

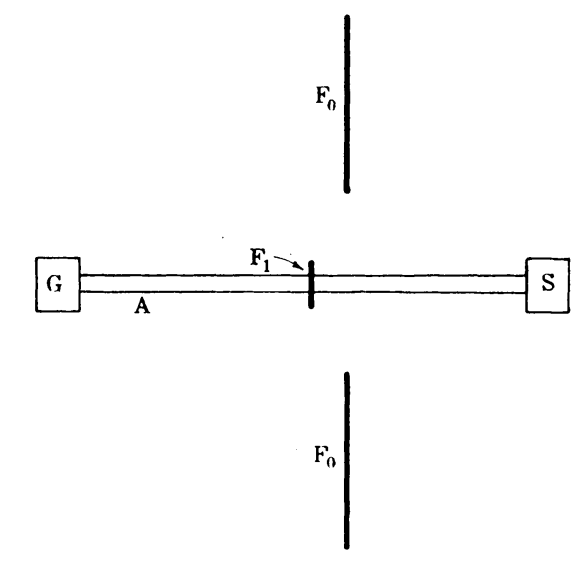

FIG. 2

The complete shape of the energy front by (1), (2) and (3) thus is like Fig. 5. That is, perpendicular at some distance $F_{0}$ from the conductor $A$, and bent

1. From the velocity of light $1 / \sqrt{L C}=3 \times 10^{10} \mathrm{~cm}$. per sec. by the factor

$$
\sqrt{1-\frac{4 r^{2}}{z^{2}}}
$$

where $z=\sqrt{L / C}$ is the surge impedance of the circuit and $L$, $C$ and $r$ the inductance, capacity and resistance per unit length. 
back nearer to the conductor, at $F_{1}$ as well as further away from the conductor at $F_{2}$. That is, the energy flow is parallel to the conductor at some distance from it, as shown by arrow $D_{0}$; converging towards the con-

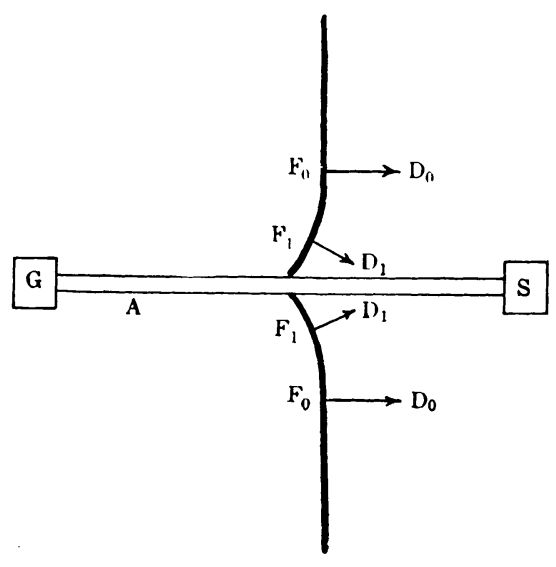

FIG. 3

ductor near it, at $D_{1}$, and diverging from the conductor at greater distance from it, $D_{2}$.

Thus far we have the viewpoint of the scientist. Next, that of the engineer comes in. Engineering is science applied to the use of man. The engineer thus resolves the phenomena into components according to their relation to man, useful or wasteful.

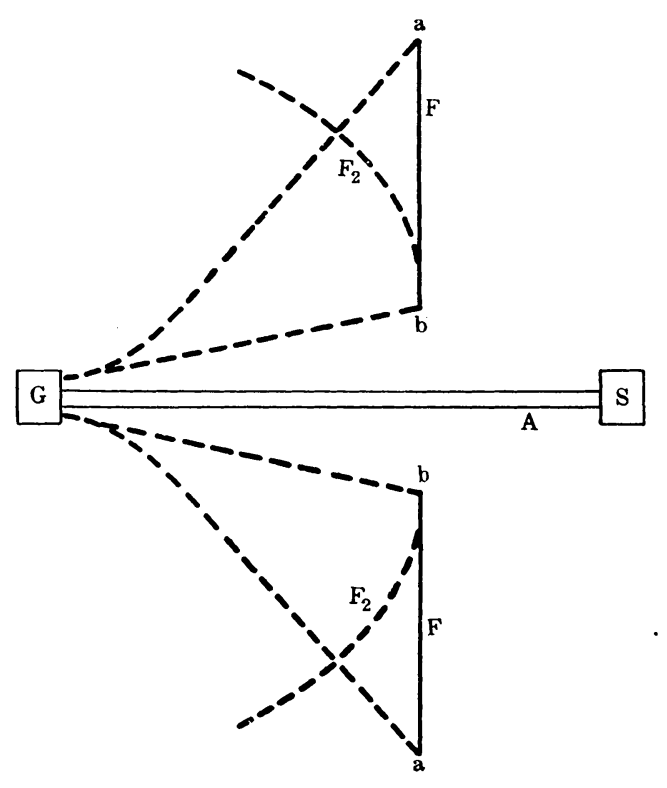

FIG. 4

Thus the energy flow in Fig. 5 may be resolved into components; those parallel with the conductor, $D_{0}$, $D_{01}, D_{02}$ of Fig. 6, and those perpendicular to the conductor $D_{1}{ }^{\prime}$ and $D_{2}{ }^{\prime}$. The former give the energy which arrives at the receiving station $S$, the useful energy for which the conductor was erected ${ }^{2}$. The latter give the energy which does not arrive at its intended destination, but is wasted; absorbed by the conductor $A$ and dissipated as heat: $D_{1}{ }^{\prime}$, or scattered as radiation through space $D_{2}{ }^{\prime}$.

We separate the two radial components, since their economic relation to us is different. The inward component $D_{1}{ }^{\prime}$ is responsible for the amount of conductor material required, the outward component $D_{2}{ }^{\prime}$ does not require line copper, but may lead to interference with other circuits. Furthermore, in other circuits, other ones of the components of energy flow may be useful or wasteful. Thus in the antenna circuit of radio communication, the radially outward component $D_{2}{ }^{\prime}$ is the useful energy which carries the message, while the radially inward component $D_{1}{ }^{\prime}$ is waste energy.

The next step for the engineer is to calculate sepa-

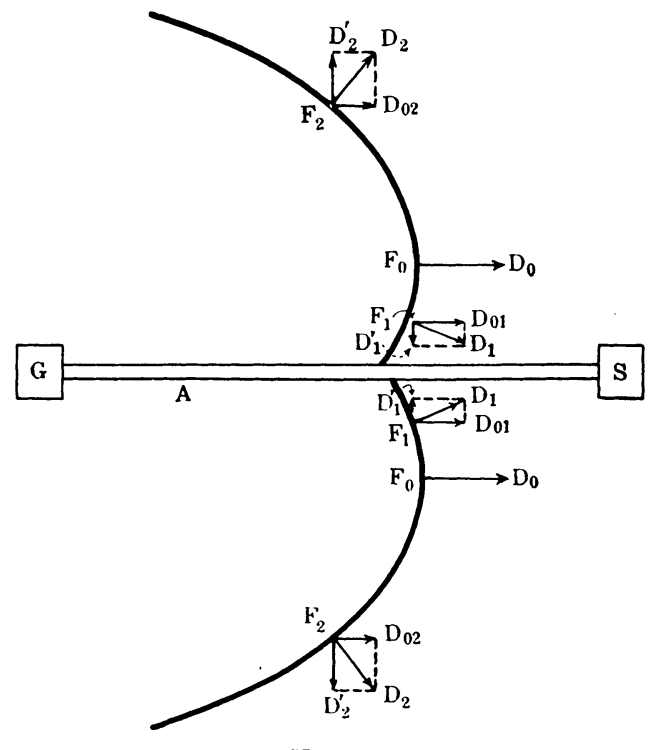

Fig. 5

rately the components, so as to make the useful ones large, the wasteful ones small.

Thus in calculating the radiation component $D_{2}{ }^{\prime}$, we consider only the radially outward energy flow $D_{2}{ }^{\prime}$, but disregard the parallel flow $D_{0}$ and $D_{02}$, that is, consider the current phase as constant throughout the conductor $A$, and the conductor thus is the radiator.

It is obvious that ultimately the radiated energy as well as the transmitted energy and the energy dissipated as heat in the conductor come from the generating station $G$.

Under transmission line conditions at machine frequencies, the radiation component is negligible, so that Fig. 3 sufficiently represents the energy wave; $D_{2}{ }^{\prime}$ becomes the dominating component however in radio communication.

2. This useful component of energy flow we may again divide into two components, the one which remains at the receiving station, the true power; and the one which oscillates between receiving station and generating station, the reactive power. 